\title{
Procuring healthcare public-private partnerships (PPPs) through unsolicited proposals during the COVID-19 pandemic
}

\author{
Carter B. Casady ${ }^{1 *}$ and David Baxter ${ }^{2}$
}

\section{Structured Abstract}

Purpose

The novel coronavirus (COVID-19) pandemic has left nations around the world scrambling to procure emergency healthcare capacity, services, and equipment. To meet this unprecedented demand on global healthcare systems, governments are increasingly looking to partner with the private sector via public-private partnerships (PPPs). However, the protracted procedures of traditional PPP procurements are not suitable for times of crisis. This is where unsolicited proposals (USPs) may play a pivotal role.

\section{Design/methodology/approach}

In order to explore the relevance of USPs for the current pandemic, this Viewpoint article describes both the advantages and challenges of USPs, discusses the emergence of several PPPs to combat COVID-19 as well as some of the ad hoc processes governing current USP consideration, highlights an example of streamlined USP solicitation from Pennsylvania's Department of Transportation (PennDOT), and articulates a pragmatic and practical approach for encouraging and procuring healthcare USPs.

\section{Findings}

This Viewpoint article concludes that USPs could play a crucial role in the COVID-19 pandemic as boundary spanners between public agencies and the private sector in the PPP procurement process.

\section{Societal Implications}

Deploying proactive and strategic healthcare PPPs at speed and scale through digital USP platforms may help mitigate the pandemic's long-term effects. Digital USP platforms may also serve as crucial tools for effective crisis communication, decision-making, and partnership.

\section{Originality}

Using the digital USP platforms proposed in this article, infrastructure organizations can develop and maintain effective partnerships with other sector organizations prior to and during crises like COVID-19.

Keywords: novel coronavirus (COVID-19); public-private partnerships (PPPs); unsolicited proposals (USPs)

\footnotetext{
${ }^{1}$ Bartlett School of Sustainable Construction, University College London, London, UK; c.casady@ucl.ac.uk, *Corresponding author

${ }^{2}$ Infrastructure Development/PPP Consultant and Steering Committee Member for the World Association of PPP Units \& Professionals (WAPPP), dbaxter@wappp.org
} 


\section{Introduction}

As the novel coronavirus (COVID-19) continues to ravage communities across the globe, national, state, and local governments are increasingly encouraging the private sector to develop solutions that will mitigate the pandemic's effects. In this COVID-19 epoch, new forms of public-private partnerships (PPPs) are being contemplated by political, economic, and healthcare leaders, both philanthropic and conventional contractual arrangements between the public and private sector that bundle multiple phases of the project lifecycle-i.e. design, construction, financing, operations, and maintenance (WBG 2017a; Casady et al. 2018, 2020; Casady 2020). While the news is filled with uplifting stories of corporations deploying their resources and supply chains to address systemic shortages in personnel protective equipment (PPE), medical devices, and other healthcare capacity, there remains a lack of coordinated and strategic initiatives for formal, longterm partnerships with the private sector (Baxter 2020a). Although the World Health Organization (WHO) has drafted interim guidance encouraging governments to "adopt a whole-of-government and whole-of-society approach in responding to the COVID-19 pandemic" which includes enhanced private sector engagement and mobilization (Hanlon, Hellowell, Eldridge, and Clarke 2020, p. 1), ${ }^{3}$ the procurement of traditional healthcare PPPs is often a protracted process involving multiple steps - i.e. expressions of interest, requests for qualifications, requests for proposals, bidder selection, and commercial/financial close. Under normal circumstances, this procurement method is preferred and mandated to ensure competitiveness and transparency. Unfortunately, these are not normal times. This is where unsolicited proposals (USPs) may play a pivotal role in the COVID-19 crisis.

Although USPs continue to be a controversial topic of policy discussion, the academic literature still inexplicably lacks information on this topic. This Viewpoint article thus aims to offer a pragmatic and practical approach for USP healthcare procurements aimed at combatting COVID-19. We begin by succinctly describing why USPs are advantageous in the COVID-19 crisis. Next, we outline the practical challenges and limitations of USPs in practice. Then, we highlight the emergence of several PPPs to combat COVID-19 as well as some of the ad hoc processes governing current USP consideration. This leads into our case example of streamlined USP solicitation from Pennsylvania's Department of Transportation (PennDOT). Subsequently, we articulate a pragmatic and practical approach to healthcare USPs using digital platforms. Finally, we conclude by reflecting on the role USPs could play in the COVID-19 pandemic as crucial boundary spanners between public agencies and private sector in the PPP procurement process.

\section{Why Unsolicited Proposals (USPs)?}

In general terms, unsolicited proposals (USPs) are proposals submitted by private firms to public sector agencies without prior solicitation or request (Hodges and Dellacha 2007; Marques 2018; Osei-Kyei and Chan 2021). Popular in several developed and developing countries, USPs usually serve as:

an alternative to the traditional project initiation method where the private sector, rather than the government, takes the leading role in identifying and developing a project. In practice, many public authorities across the world resort to USPs motivated by the

\footnotetext{
${ }^{3}$ WHO's guidance highlights three tiers of public-private interaction.
} 
perspective of solving the challenges brought by their lack of capacity to identify and develop projects (WBG 2017b, p. v).

Around the world, it is not uncommon for the public sector to consider potential PPP projects through USPs (WBG 2018). For instance, the World Bank's Private Participation in Infrastructure (PPI) Database indicates "approximately 4 percent of infrastructure projects within . . low- and middle-income economies are started at the initiative of a private sector entity without an explicit request from a government to do so" (WBG 2018, p. 62). Osei-Kyei et al. (2018b, p. 221) stress that government interest in USPs tends to stem from enhanced private sector innovation, the absence of public sector capacity to "identify, prioritise and procure projects," limited investment/development interests in remote areas, and "rapid implementation of PPP projects."

Unlike typical PPPs which are prone to longer tendering durations and higher transaction costs than traditional public provision (Casady et al. 2019; HM Treasury 2012; Soliño and Gago de Santos 2010; NAO 2007; Ahadzi and Bowles 2004), unsolicited PPP proposals can also offer expedited procurement timelines. Although little robust research exists to capture the differences between proposal mode (Yun et al. 2015), countries find expedited procurement to be an attractive feature of USPs. For example, in Colombia, privately financed USPs can "take advantage of an abbreviated procurement process, suggesting that the government has intentionally structured its USP framework such that these USP projects can be implemented more rapidly" (World Bank 2017 e, p. 24). This is an important consideration because long tendering periods can impact public sector investment efficiency (CCPPP 2015; KPMG 2010), delay the completion of projects, and impose higher social costs on citizens who are unable to access critical public services (HM Treasury 2010).

Overall, governments may be motivated to use USPs because they "solve certain structural concerns - such as the slow implementation of publicly initiated projects — or address identified gaps in publicly initiated and developed projects - such as lack of innovation" (World Bank 2017e, p. 22). By overcoming a lack of public-sector capacity and harnessing private-sector innovation and creativity, USPs may thus be able to offer critically needed healthcare solutions during the COVID-19 pandemic through shortened bidding and contract award procedures while contributing to national infrastructure goals (Hodges and Dellacha 2007).

\section{The Challenges of USPs}

Although "this procurement option can indeed be useful and valuable[,]" Marques (2018, p. 435) notes that USPs "should be utilized with caution." This is because USPs face their own institutional challenges. Carbonara, Costantino, and Pellegrino (2016, p. 491) stress that the design and choice of tendering procedures can be influenced by a variety of other factors, such as "the number of bidders, the project size, the project complexity, and the institutional environment." Abdel Aziz and Nabavi (2014) also highlight that companies avoid USPs because of government mismanagement, transparency problems, and the lack of competition, along with inadequate guidelines and short bidding times. In many countries, USPs are generally avoided because they can divert public resources away from other government strategic plans and priorities, fail to attract competition, and create opportunities for corruption (WGB 2017b). Although World Bank Group's (2018) analysis of PPP procurement for 135 economies suggests a significant number of economies explicitly permit (57 percent) or prohibit USPs (3 percent), 40 percent do not regulate 
them at all. This lack of USP regulations "can [generally] be construed as a prohibition in practice, since PPP procurement methods are limited to those expressly regulated" (WBG 2018, p. 64). ${ }^{4}$

Nonetheless, USPs still take place in practice in a sizable number of countries that do not regulate them (i.e. 10 percent of the total). ${ }^{5}$ In these instances, the absence of clear regulatory frameworks for USPs may enable private sector actors to circumvent procurement rules established by public sector institutions. Casady (2020, p. 15) suggests that "countries prioritizing the political and governance strengths of PPPs may [already] be exploiting the absence of external transparency - i.e. the extent to which internal information is visible to the outside world - in order to deliver PPP projects."

Unsolicited proposals (USPs) thus need to be properly regulated to prevent nontransparent behavior. Osei-Kyei et al. (2018a) asserts there are seven critical strategies for managing these types of proposals. These strategies include:

1) establishing well-structured and clear USP policy guidelines,

2) conducting thorough assessments of value for money, innovation, cost, and risks,

3) employing highly skilled and competent staff for proposal evaluations,

4) executing a fair, competitive, and transparent tendering process,

5) engaging in extensive public consultation and stakeholder outreach,

6) evaluating USP impacts on sector/national policy, and

7) protecting the intellectual property rights of the original proponent.

Above all, the public sector must have the capacity to technically validate proposals and align them with national strategic interests and/or attempts to comply with Sustainable Development Goal (SDG) commitments (Zawawi, Kulatunga, and Thayaparan 2016). Otherwise, governments run the risk of pursuing projects that yield low Value-for-Money (VfM). When a meritorious project does arise, governments might consider opening up the USP to a competitive and transparent procurement procedure (e.g. using the "Swiss" method) where the original bidder is given some advantages - i.e. extra project evaluation points or the right to make a final counter offer — during the process. Moreover, additional proponents should be given ample time to compete in a USP procedure because "providing a short period for competing bidders to submit bids . . . limits competition" (WBG 2018, p. 67). USPs in the COVID-19 epoch thus still "require rather, aggressive management by a strong, competent government" in order to ensure adequacy, transparency, and fairness (Kettl, 2011, p. 6).

\section{USPs to Combat COVID-19}

Although many countries remain generally averse to USPs, the COVID-19 pandemic has prompted governments around the world to rapidly form ad hoc partnerships with the private sector. For example, at the outset of the pandemic, the Australian government engaged the private sector to shore up bed facilities and boost medical staff in hospitals. The agreement secured 30,000 hospital beds and 105,000 nurses and staff to help fight the outbreak. The government additionally offered "agreements to 657 private and not-for-profit hospitals to ensure their viability, in return for maintenance and capacity during the COVID-19 response" (Khaliq 2020). Other somewhat unprompted partnerships have also been used to develop COVID-19 tests in Iceland, deploy contract tracing apps in India, and support vaccine development in the European Union

\footnotetext{
${ }^{4}$ This is the case in Canada, the United Kingdom, and most other EU economies.

${ }^{5}$ This percentage is the highest in East Asia (20 percent).
} 
(Korchakova-Heeb 2020). Likewise, the U.S. federal government has formed several partnerships with industry and academia to combat the crisis, including a "partnership with national pharmacy and grocery retail chains . . . to seamlessly provide Americans convenient access to COVID-19 testing" (HHS 2020b), a COVID-19 High Performance Computing Consortium6 to "provide access to the world's most powerful high-performance computing resources in support of COVID19 research" (COVID-19 HPC Consortium 2020), and the Accelerating COVID-19 Therapeutic Interventions and Vaccines (ACTIV) PPP, an initiative intended "to accelerate the development, manufacturing, and distribution of COVID-19 vaccines, therapeutics, and diagnostics" (HHS 2020a).

Similar partnerships have also emerged at the international level. For instance, Gavi, the Vaccine Alliance - a global healthcare PPP founded to increase access to immunizations in poor countries - recently deployed an innovative financing instrument (Gavi COVAX AMC) to support the participation of 92 low- and middle-income economies in the COVAX Facility, "a global risksharing mechanism for pooled procurement and equitable distribution of eventual COVID-19 vaccines" (Gavi 2020). Other initiative likes the Access to COVID-19 Tools (ACT) Accelerator have:

connect[ed] the European Commission with the Bill \& Melinda Gates Foundation (the Gates Foundation); CEPI; Gavi, the Vaccine Alliance (Gavi); The Global Fund to Fight AIDS, Tuberculosis and Malaria (The Global Fund); Unitaid, Wellcome Trust, and the World Health Organization (WHO) to craft a global pandemic response (NASEM 2020).

Even at the local level, USPs are being encouraged in response to the health crisis. For instance, the Los Angeles County Metropolitan Transportation Authority (LA Metro) has prioritized USPs "related to pandemic response, service relaunch, and regional recovery that support efficient mobility in a changed world," specifically innovations aimed at enhancing cleaning and hygiene, maintaining social distancing, relaunching service to meet residual demand/emerging needs, and reducing costs or generating revenue as Metro adapts and evolves over the course of the pandemic. However, "[d]ue to the dynamic and uncertain nature of the crisis, and to aid prioritization, Metro has temporarily suspended its 90-day review period," leaving previously submitted USPs to be considered on a case-by-case basis (Borgman 2020). Relying on such a make-do process to manage the many competing needs of government is ultimately unsustainable, especially in times of crisis.

\section{Adopting Streamlined USP Procedures: The Case of PennDOT}

Fortunately, some government institutions have realized the value of managing USPs in a wellordered manner. An outstanding example of streamlined USP solicitation comes from Pennsylvania's Department of Transportation (PennDOT), located in the United States. PennDOT hosts a website dedicated to encouraging unsolicited proposals. ${ }^{7}$ However, PennDOT's USP solicitation and procurement process is not an ad hoc procedure. PennDOT has several conditions in place to ensure that its procurement team is not overwhelmed by frivolous USPs and distracted from its planned pipeline of traditional/PPP projects. These USP guidelines are harmonized within PennDOT's PPP-enabling legislation (Act 88) and PPP implementation manual. Measures that have been set in place to streamline and filter unsolicited proposals include:

\footnotetext{
${ }^{6}$ See https://covid19-hpc-consortium.org/.

${ }^{7}$ The PennDOT USP website can be found here:

https://www.penndot.gov/ProjectAndPrograms/p3forpa/Pages/Unsolicited-Proposals.aspx
} 
- accepting USPs only twice a year (i.e. July and October);

- preregistering potential private partners before proponents can submit proposals, including prescribed qualifications; and

- outlining a list of desirable/acceptable project types (and sectors) that would be considered by PennDOT.

Once the proposals are submitted, a seven-member Public Private Transportation Partnership Board examines and approves potential projects. According to PennDOT (2019), "if the board determines a state operation would be more cost-effectively administered by a private company, the company will be authorized to ... enter into a contract to either completely or partially take over that operation for a defined period of time." This process allows the public sector to solicit, technically evaluate, and procure USPs in a systematized manner which offers serious and qualified private sector partners a competitive platform to submit innovative ideas. Additionally, this structured application process goes a long way to appease any concerns the public may have about the corruption of traditional procurements, thereby enhancing the legitimacy of such undertakings (Casady 2020; Casady et al. 2020). By introducing qualification criteria and specific project needs, PennDOT has also introduced measures that safeguard its institutional capacity, prioritize/align its strategic objectives, and strengthen the VfM case for PPPs (Baxter 2020c). Moreover, progressive public sector institutions who adopt a PennDOT-type approach can build in other project qualification criteria to account for environmental and "People First PPP" considerations, as proposed by the UNECE PPP Center of Excellence in Geneva (UNECE 2019). ${ }^{8}$

\section{A Pragmatic and Practical Approach to Healthcare USPs: A Digital Platform}

Moving forward, governments are expected to increasingly seek partnerships with the private sector to manage the short-, medium-, and long-term fallout stemming from the COVID-19 pandemic (Baxter and Casady 2020). These partnerships could be more effectively initiated, evaluated, and procured using a collaborative approach that encourages qualified partners to participate competitively in transparent procurements. A digital platform similar to PennDOT's may help streamline the current chaotic, unorganized, and ad hoc processes governments have relied on to date to manage this crisis. Such a platform, along with complementary governance arrangements, would also encourage the private sector to share innovations with the public sector in a controlled and organized manner, thereby by bolstering legitimacy, trust, and capacity for emergency healthcare USPs. Additionally, digital USP platforms - initiated at a national or international level - would ensure that both nations and states do not unnecessarily compete for the same resources. Ultimately, these platforms could serve as a gateway for sustainable and resilient procurements of healthcare infrastructure, services, pharmaceuticals, and equipment.

Although digital USP platforms might not be deployable imminently, these tools offer a pragmatic and practical approach for medium- and long-term strategic healthcare procurements. In the post COVID-19 recovery process, these platforms may serve as an accessible "innovation gateway" that harmonizes national (and even international) crisis response priorities. Systematized USP procurements would also more likely result in sustainable and resilient projects that protect the public interest, generate VfM, conform with public fiscal priorities, reflect fair market prices/risk allocation, promote transparency and accountability, and align with existing PPP

\footnotetext{
${ }^{8}$ See https://www.uneceppp-icoe.org/people-first-ppps/ for more details.
} 
guidelines (WBG, 2017b). Additionally, national and international platforms of this nature would strengthen the global efforts of healthcare partners if COVID-19 continues to resurge or another pandemic materializes in the future. Overall, harmonized digital platforms would most certainly allow healthcare providers to efficiently identify acceptable USPs supporting pandemic mitigation, containment, and future prevention efforts.

\section{Prerequisites for the Adoption of Digital USP Procurement Platforms}

Naturally, the adoption and effectiveness of any USP procurement platform will be influenced by the broader institutional environment and political setting. Governments developing USP procedures must therefore ensure their processes are complemented by an effective PPP regulatory regime as well as strong institutional capacity governing the development and implementation of both publicly and privately initiated PPPs (WBG, 2017b). At the outset, governments will also need to define high-level parameters of their USP processes, including the objectives, scope, and guiding principles. Healthcare PPP procurements using a USP approach based on the PennDOT model would thus need to meet the following prerequisites:

1. a streamlined and harmonized procedure that avoids unnecessary bureaucracy and sets clear procurement objects/deliverables for all relevant stakeholders;

2. a user-friendly digital platform that allows new private sector partners competitive access to a procurement mechanism that allows them to introduce innovative ideas and solutions for desperately needed healthcare works, services and supplies;

3. the establishment of a pool of qualified partners who can implement actions that have significant impacts (inclusive of international contractors who might be better positioned than local contractors); and

4. an established set of achievable parameters for VfM and People First PPPs.

Of course, these prerequisites would naturally be context-specific and tailored to both national strategic interests as well as SDG commitments. More importantly, these prerequisites would be embedded within broader considerations of each phase in the USP process-i.e. submission, evaluation, project development, and procurement (see figure 1).

\section{[insert figure 1 about here]}

This process flow, developed from international best practices at the World Bank Group (2017a, 2017b, 2017c), serves as an ideal blueprint for digital USP platforms, one that illustrates how such platforms can serve as crucial boundary spanners between public agencies and private sector in the PPP identification and screening phase of the procurement process. After all, Kapucu (2006, p. 207) stresses that information technologies, like a digital USP platform, are crucial for "achiev[ing] effective communication and decision-making goals in emergencies."

\section{Conclusion}

We are running out of time to solicit and procure emergency healthcare capacity, services, and equipment to combat the ongoing COVID-19 pandemic. Under normal circumstances, traditional procurement of additional healthcare PPPs would be ideal for meeting the unprecedented strain placed on global healthcare systems. However, these are not normal times and speed of delivery is crucial for saving lives. In this light, unsolicited proposals (USPs) can play a pivotal role in 
delivering emergency healthcare PPPs during the COVID-19 crisis. Using a digital USP platform, organizations [could] develop and maintain effective partnerships with other sector organizations prior to [and during] emergencies" (Kapucu, 2006, p. 211). Figure 2 illustrates how such USP platforms would broadly serve as boundary spanners in the procurement of healthcare PPPs.

[insert figure 2 about here]

Moving forward, governments looking to develop USP policies and processes for the COVID-19 pandemic can leverage the skills of the global PPP practitioner community and collaborate with professional organizations such as the World Association of PPP Units \& Professionals (WAPPP), ${ }^{9}$ the International Sustainable Resilience Center (ISRC), ${ }^{10}$ and PPPHealth4All. ${ }^{11}$ However, governments should also be cautious and not get "transfixed by the PPP ideal" (Hodge, Greve, and Biygautane, 2018, p. 1109). Instead, public agencies should reflect on innovative ways to reorganize their policy-making processes and existing institutional structures to most effectively combat the COVID-19 pandemic in a sustainable and resilient manner.

\section{References}

Abdel Aziz, Ahmed, and Human Nabavi. 2014. "Unsolicited proposals for PPP projects: private sector perceptions in the USA." In Construction Research Congress 2014: Construction in a Global Network, pp. 1349-1358.

Ahadzi, Marcus, and Graeme Bowles. 2004. "Public-private partnerships and contract negotiations: an empirical study." Construction Management and Economics 22, no. 9: 967978.

Baxter, David. 2020a. "How will coronavirus affect public-private partnerships?" World Bank Blogs.

Baxter, David. 2020b. "The Need for Sustainable PPP Recovery Strategies in a Post-COVID-19 World." LinkedIn.

Baxter, David. 2020c. "Pragmatic Procurements (Solicitations) of Emergency Healthcare Unsolicited Proposals During the COVID-19 Pandemic." Public Spend Forum.

Baxter, David and Carter B. Casady. 2020. "Proactive and Strategic Healthcare Public-Private Partnerships (PPPs) in the Coronavirus (COVID-19) Epoch." Sustainability 12, no. 12: 5097.

Borgman, Nolan. 2020. Updated unsolicited proposal guidance during COVID-19 health crisis. Los Angeles, CA: Los Angeles County Metropolitan Transportation Authority (LA Metro).

Carbonara, Nunzia, Nicola Costantino, and Roberta Pellegrino. 2016. "A transaction costs-based model to choose PPP procurement procedures." Engineering, Construction and Architectural Management 23, no. 4: 491-510.

Casady, Carter B. 2020. "Examining the Institutional Drivers of Public-Private Partnership (PPP) Market Performance: A Fuzzy Set Qualitative Comparative Analysis (fsQCA)." Public Management Review.

Casady, Carter, Darragh Flannery, R. Richard Geddes, Dónal Palcic, and Eoin Reeves. 2019. "Understanding PPP tendering periods in Canada: a duration analysis." Public Performance \& Management Review 42, no. 6: 1259-1278.

\footnotetext{
${ }^{9}$ See https://www.wappp.org/.

${ }^{10}$ See https://ippprc.org/.

${ }^{11}$ See https://www.pppinitiative4coe-healthcare.org/.
} 
Casady, Carter B., Kent Eriksson, Raymond E. Levitt, and W. Richard Scott. 2018. "Examining the State of Public-Private Partnership (PPP) Institutionalization in the United States." Engineering Project Organization Journal 8, no. 1: 177-198.

Casady, Carter B., Kent Eriksson, Raymond E. Levitt, and W. Richard Scott. 2020. “(Re)Defining Public-Private Partnerships (PPPs) in the New Public Governance (NPG) Paradigm: An Institutional Maturity Perspective." Public Management Review 22, no. 2: 161-183.

Canadian Council for Public-Private Partnerships (CCPPP). 2015. Public-private partnerships: What the world can learn from Canada. Toronto, Canada: The Canadian Council for PublicPrivate Partnerships.

COVID-19 HPC Consortium. 2020. Accessed at: https://covid19-hpc-consortium.org/.

Gavi. 2020. What is the Gavi COVAX AMC? Washington, DC: Gavi, the Vaccine Alliance.

Hanlon, Barbara O., Mark Hellowell, Cynthia Eldridge, and David Clarke. 2020. "An Action Plan to Engage the Private Sector in the Response to COVID-19 $\mid$ Interim Guidance." Geneva, Switzerland: World Health Organization (WHO).

Hodges, John T., and Dellacha, Georgina. 2007. Unsolicited Infrastructure Proposals How Some Countries Introduce Competition and Transparency. World Bank, Working Paper 1 (PPIAF).

U.S. Department of Health and Human Services (HHS). 2020a. Fact Sheet: Explaining Operation Warp Speed. Washington, DC: HHS.

U.S. Department of Health and Human Services (HHS). 2020b. HHS Extends COVID-19 Testing Public-Private Partnership. Washington, DC: HHS.

HM Treasury. 2010. HM treasury review of competitive dialogue. London, UK: HM Treasury. HM Treasury. 2012. A new approach to public private partnerships. London, UK: HM Treasury. Hodge, Graeme, Carsten Greve, and Mhamed Biygautane. 2018. "Do PPP's work? What and how have we been learning so far?." Public Management Review 20, no. 8: 1105-1121.

Kapucu, Naim. "Interagency communication networks during emergencies: Boundary spanners in multiagency coordination." The American Review of Public Administration 36, no. 2 (2006): 207-225.

Kettl, Donald F. 2011. Sharing Power: Public Governance and Private Markets. Washington, DC: Brookings Institution Press.

Khaliq, Riyaz. 2020. "Australia: Public-private partnership to fight virus." Anadolu Agency. Korchakova-Heeb, Natalia. 2020. "\#PPPsAgainstCorona | Public-Private Partnerships in the time of Corona." Inaugural webinar for Africa Public Private Partnerships Network (AP3N), June 10, 2020.

KPMG. 2010. PPP procurement: Review of barriers to competition and efficiency in the procurement of PPP projects. Sydney, Australia: KPMG Corporate Finance.

Marques, Rui Cunha. 2018. "Empirical evidence of unsolicited proposals in PPP arrangements: A comparison of Brazil, Korea and the USA." Journal of Comparative Policy Analysis: Research and Practice 20, no. 5: 435-450.

National Academies of Sciences, Engineering, and Medicine (NASEM). 2020. Public Private Partnership Responses to COVID-19 and Future Pandemics: Proceedings of a Workshop in Brief. Washington, DC: The National Academies Press.

National Audit Office. 2007. Improving the PFI tendering process. Report by the Comptroller and Auditor General; HC 149 Session 2006-2007. London: HMSO. 
Osei-Kyei, Robert, and Albert PC Chan. 2021. "Management of Unsolicited Public-Private Partnership Projects". In International Best Practices of Public-Private Partnership, edited by Robert Osei-Kyei and Albert PC Chan, p 109-126. Springer, Singapore.

Osei-Kyei, Robert, Albert PC Chan, Ayirebi Dansoh, Joseph K. Ofori-Kuragu, and Goodenough D. Oppong. 2018a. "Strategies for effective management of unsolicited public-private partnership proposals." Journal of Management in Engineering 34, no. 3: 040180.

Osei-Kyei, Robert, Albert PC Chan, Ayirebi Dansoh, Joseph Kwame Ofori-Kuragu, and Emmanuel Kingsford Owusu. 2018b. "Motivations for adopting unsolicited proposals for public-private partnership project implementation." Journal of Financial Management of Property and Construction 23, no. 2: 221-238.

Pennsylvania Department of Transportation (PennDOT). 2019. "PennDOT Accepting Unsolicited Public-Private Partnership Proposals Until October 31." Harrisburg, PA: Commonwealth of Pennsylvania.

Soliño, Antonio Sánchez, and Pilar Gago de Santos. 2010. "Transaction costs in transport publicprivate partnerships: comparing procurement procedures." Transport Reviews 30, no. 3: 389406.

World Bank Group (WBG). 2017a. Public Private Partnerships Reference Guide: Version 3. Washington, DC: International Bank for Reconstruction and Development/World Bank.

World Bank Group (WBG). 2017b. Policy Guidelines for Managing Unsolicited Proposals in Infrastructure Projects |Volume 1: Main Findings \& Recommendations. Washington, DC: Public-Private Infrastructure Advisory Facility (PPIAF).

World Bank Group (WBG). 2017c. Policy Guidelines for Managing Unsolicited Proposals in Infrastructure Projects |Volume 2: Guidelines for the Development of a Policy for Managing Unsolicited Proposals in Infrastructure Projects. Washington, DC: Public-Private Infrastructure Advisory Facility (PPIAF).

World Bank Group (WBG). 2017d. Policy Guidelines for Managing Unsolicited Proposals in \begin{tabular}{l|l} 
Infrastructure Projects & Volume 3: Review of Experiences with Unsolicited Proposals in
\end{tabular} Infrastructure Projects. Washington, DC: Public-Private Infrastructure Advisory Facility (PPIAF).

World Bank Group (WBG). 2017e. Review of Experiences with Unsolicited Proposals in Infrastructure Projects. Washington, DC: Public-Private Infrastructure Advisory Facility (PPIAF).

World Bank Group (WBG). 2018. Procuring Infrastructure Public-Private Partnerships Report 2018: Assessing Government Capability to Prepare, Procure, and Manage PPPS. Washington, DC: International Bank for Reconstruction and Development/World Bank.

United Nations Economic Commission for Europe (UNECE). 2019. "What are People-First PPPs?" Geneva, Switzerland: UNECE International Center of Excellence.

Yun, Sungmin, Wooyong Jung, Seung Heon Han, and Heedae Park. 2015. "Critical organizational success factors for public private partnership projects-a comparison of solicited and unsolicited proposals." Journal of Civil Engineering and Management 21, no. 2: 131-143.

Zawawi, Muhammad Imran Zin, Udayangani Kulatunga, and Menaha Thayaparan. 2016. "Malaysian experience with public-private partnership (PPP): Managing unsolicited proposal." Built Environment Project and Asset Management 6, no. 5: 508-520 


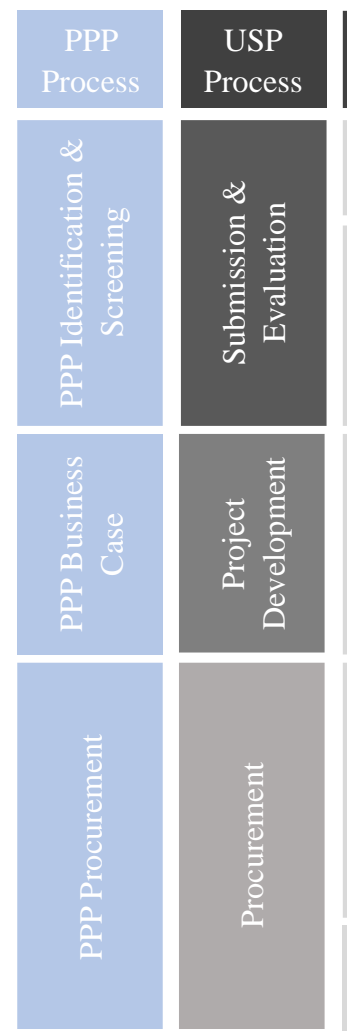

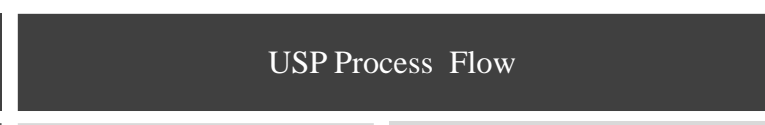

Proponent submits USP to public agency

Public agency may request clarifications from proponen

The public agency:

- Verifies USP meets evaluation criteria

- Requests evidence of qualifications (if relevant)

- Uses benchmarking / market testing (if relevant) to evaluate project

- Discloses relevant documentation

- Determines most appropriate development / procurement method

The public agency:

- (1) undertakes project development with its external advisors, or (2) signs a project-development agreement with proponent

- Uses benchmarking / market testing (if relevant) to evaluate project

- Discloses relevant documentation

- Confirms most appropriate procurement method

To prepare for procurement, the public agency:

- Secures right-of-way and/or acquires land

- Obtains environmental and social clearance

- Develops a draft PPP contract (together with external advisors)

- If competitively procuring, develops draft procurement documentation

- If preparing for a direct negotiation, signs the direct-negotiation

protocol

- Uses benchmarking and market testing (if necessary)

The public agency either: (1) undertakes a competitive tender, or (2) directly negotiates the PPP contract with the proponent according to the

direct-negotiation protocol

\section{USP Approvals \\ COMPLIANCE CHECK \\ Public agency checks whether the USP submission is compliant \\ PROJECT EVALUATION \\ Public agency verifies if USP meets evaluation criteria}

DEV \& PROCUREMENT DECISION

Decision-making authority selects project

development and procurement method

PROJECT APPROVAL

Decision-making authority determines whether the project should proceed to procurement

PROCUREMENT APPROVAL

Decision-making authority approves procurement documentation

CONTRACT AWARD

Decision-making authority awards the PPP contract

Source: WBG (2017b)

Figure 1: USP Process Flow and Key Considerations

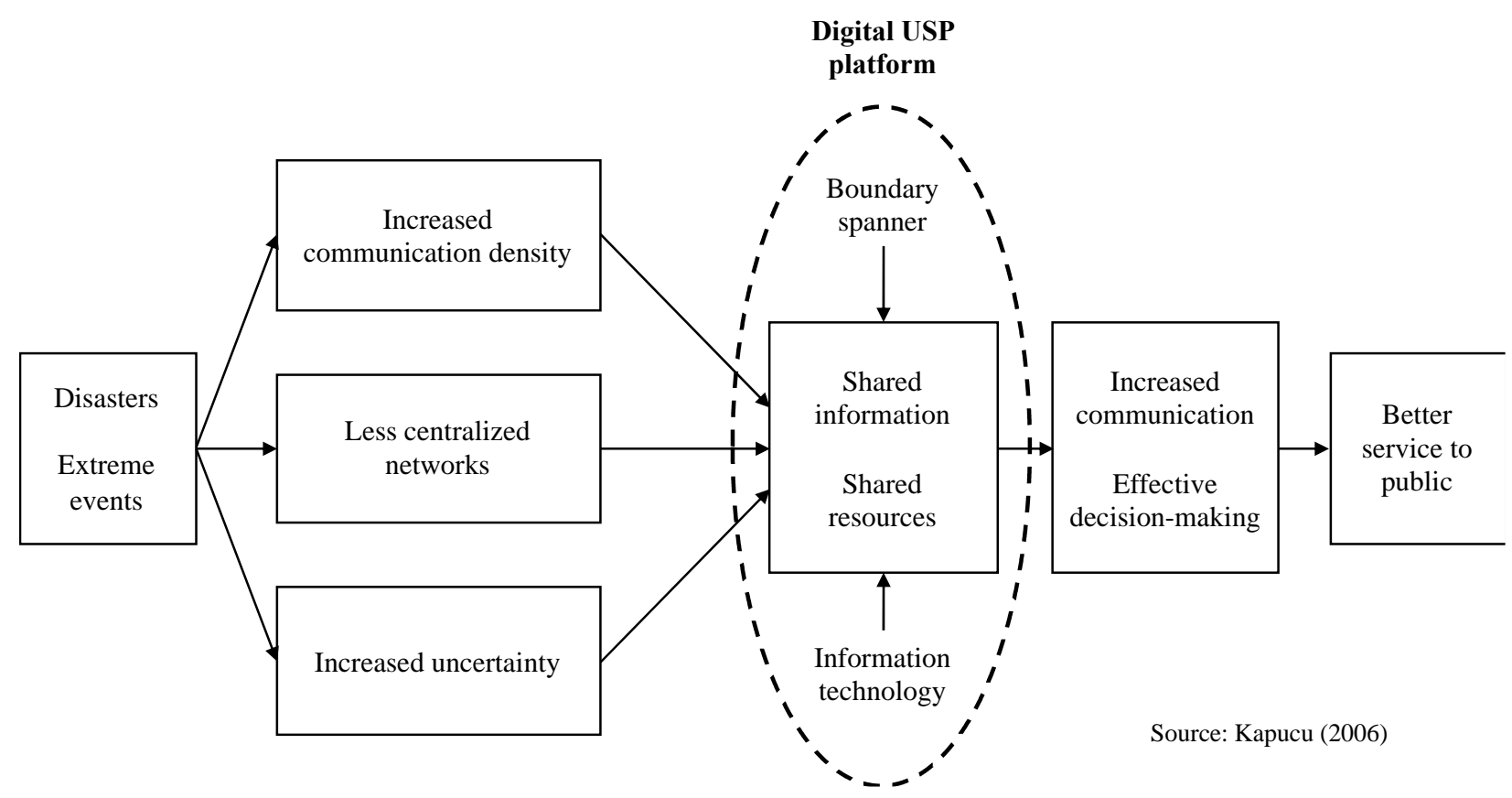

Figure 2: Emergency Communication and Coordination Schematic 